\title{
Bayesian regions
}

\author{
of evidence \\ (for normal distributions)
}

\section{Michael Höfler $^{1}$}

1: Clinical Psychology and Behavioural Neuroscience, Institute of Clinical Psychology and Psychotherapy, Technische Universität Dresden, Dresden, Germany

Corresponding author: Michael Höfler, Chemnitzer Straße 46, Clinical Psychology and Behavioural Neuroscience, Institute of Clinical Psychology and Psychotherapy, Technische Universität Dresden, 01187 Dresden, Germany. michael.hoefler@tu-dresden.de, +49 35146336921

Submitted to Meta-Psychology. Participate in open peer review by commenting through hypothes is directly on this preprint. The full editorial process of all articles under review at Meta-Psychology can be found following this link: https://tinyurl.com/mp-submissions

You will find this preprint by searching for the authors name. 


\section{Abstract}

Bayesian data analysis allows a researcher to assess whether a claim about an effect (e.g. effect $>0$, effect $>\Delta, \mid$ effect $\mid<\Delta)$ ) is justified given the data and a prior distribution, expressing her or his personal belief before seeing the data. However, the recipients of the analysis might use different priors, so it remains unclear whether they would share the claim. "Reverse Bayes" analysis and the "sufficiently sceptical prior" address this problem by asking how strongly one may believe in the absence of an effect in order to be convinced otherwise by the data. A method called "Region of Evidence" is presented that takes this idea and extends it for any normal prior (and a normally distributed estimate). It visualises all the priors that, if they had been used, would support the claim, including those that favour a positive or negative effect. Since the method depends only on an estimate and its standard error, it can be easily applied to previously published results. The paper describes the method and its implementation in a new Stata command called arevi, which can be freely used and modified. 


\section{Introduction}

Bayesian methods have been extensively described and discussed (Berger \& Berger, 1985; Carlin \& Louis, 2000; Gelman et al., 2013; Greenland, 2006; Vandekerckhove, 2018; Wagenmakers et al, 2018). The Bayesian approach to science formally incorporates prior beliefs about an association or effect through a prior distribution on it. Observing data serves to update this distribution and results in an effect's posterior distribution. This distribution summarizes the information on the effect, given the prior, the data and the model that describes the data, and serves as the basis for inference about the effect when assessing whether claims like effect $>0$, effect $>\Delta$ (e.g. lower threshold for practical significance) or $\mid$ effect $\mid<\Delta$ (effect neglectable) are supported or not. Reasons for doing Bayesian analysis include:

- advantage in interpretation: as posterior probability that a hypothesis is true (conditional on the above; Greenland, 2006; but the posterior probability should be compared with the prior probability; Mayo, 2018, and the references therein).

- transparency of prior assumptions (Greenland, 2006; Wagenmakers et al., 2018)

- clarification of misconceptions about frequentist p-values (Marsman \& Wagenmakers, 2017)

- accounting for complexity (Wagenmakers et al., 2018)

- stabilization of estimates, especially in small samples with many predictors (Greenland et al., 2016a; O’Hara \& Sillanpää, 2009)

- ability to incorporate assumptions about biases in estimates (Greenland, 2005, 2006, 2009a,b). 
The posterior distribution depends infinitely on the choice of the prior. Regardless of the results of the data, a prior dominates the posterior if its variance is much smaller than the variance of the data estimate (e.g. Gelman et al., 2013). This desirably reflects the flexibility to express whatever assumption on effect direction and magnitude (and uncertainty on that expressed in a distribution), but raises the question on how to handle the resulting variation in the posterior. A researcher who conducts a Bayesian analysis must not misuse this variation to produce a desired result (Dienes, 2016) and is well advised to pre-register the prior with the entire analysis to be capable of demonstrating that its choice was not affected by the data.

Meaningful variation in the posterior arises through different researchers. First, a reviewer of an analysis might request her own prior to be used to see whether the researcher's claim on an effect also stands her prior, and she, in turn, would share the claim. While the researcher may fulfil the request before the result paper is published, recipients of the paper may still have different priors. This possibility seems common since evidence on effects often comes from studies with different methods that are differently prone to various biases (Maclure \& Schneeweiss, 2001), wherefore different studies may yield different results, and there is no objective way to combine and weight such often "ragged evidence" (Greenland, 2012) in a prior. As a result, it might be unclear for researchers with different priors whether these are consistent with a claim. A positive result (claim confirmed with the analyst's prior), for instance, might not stand several pessimistic priors. This is unwarranted for a transparent science that aims to inform the audience on the consequences of assumptions on the results, so that researchers are informed if their own assumptions would support a claim or not (Höfler et al., 2018).

The Reverse Bayesian approach addresses this problem with special priors, namely sceptical priors that are centred around 0 , expressing the belief that there is no effect. The degree of 
certainty of this assumption is expressed with the inverse variance of a prior with a normal distribution (the lower this variance, the stronger the scepticism about an effect in one direction or the other; Good, 1950, p. 80 ff.; Greenland, 2006; Held, 2020; Matthews, 2001).

In this paper, this idea is extended through the Region of Evidence, a graphical method that visualizes all priors which support a target claim, also those in favour of a positive or, respectively, a negative effect (or association). The method requires that both the prior and the data contribution (likelihood estimate) must be normally distributed. With target claim we mean statements of interest on an effect that can be expressed as an interval, especially, as in statistical hypotheses, that an effect is greater 0 , greater than $\Delta$ (a lower threshold of practical significance) or neglectable (smaller than $\Delta$ and larger than $-\Delta$ ). After clarifying some methodological grounds, the method and its application are illustrated with hypothetical data, and its implementation in Stata is described.

\section{Basic assumptions}

\section{Semi-Bayes}

The method of regions of evidence is a Semi-Bayesian method; that is, it assumes that a prior on a (target) parameter, $\beta$, an effect, can be specified irrespective of other parameters in an analytic model. An analytic model is often a regression model that includes $\beta$ but might also include other parameters like an intercept term and coefficients for confounder effects (on the outcome). These receive no priors and are thus allowed to vary freely depending on the data (as they do in frequentist estimation) (Greenland, 2006, and references therein). Besides, $\beta$ must describe a main effect that can be interpreted independently from eventually prior distributions on other parameters. (A counterexample is an interaction model where $\beta$ varies 
according to another (moderator) variable like a rare event which requires a prior for more stable estimates.)

\section{Bayesian estimation}

The method focusses on interval claims that a true $\beta$ lies in a certain interval. The Bayesian approach allows calculating the probability that the claim is true (given the data, the model describing the data and the prior) through the area under the posterior distribution across the interval.

Focussing on an interval for $\beta$ reflects the stance of Bayesian estimation, where a prior on $\beta$ is a continuous distribution of $\beta$ expressing the belief that a true effect magnitude might equal any value, and that the probabilities for ranges of values (intervals of a certain length) vary smoothly across the scale of $\beta$ (e.g. according to a normal distribution). In contrast, Bayesian testing allows a positive probability to be assigned to a scalar value of $\beta$ (e.g. 0 or $\Delta$ ), as advocated by Wagenmakers and colleagues and implemented in the software JASP (Wagenmakers et al., 2018).

\section{Normality}

Likelihood-based estimates of regression coefficients are increasingly normally distributed with increasing sample size. This applies across many kinds of regression models including generalized linear models and their extensions (Severini, 2000). A factor of interest may be dummy-coded or inverval-scaled. Then, within linear models, $\beta$ equals a group difference in an outcome or a slope (standardized or not). Within a generalized linear model, $\beta$ may equal an ln-ratio and be approximated with a normal distribution (Greenland, 2006; Matthews, 2001). 
There is large literature on the choice of priors (Carlin \& Louis, 2000; Depaoli et al., 2020; Fortuin, 2021; Gelman et al., 2013; Greenland, 2006; and references therein;). Here the focus is on describing how a normal prior might be specified. This yet allows to express many prior beliefs on a $\beta$, provided that the uncertainty distributes symmetrical around the prior expectation, $\mathrm{e}_{0}$, with tails of a normal shape. Such a prior can be chosen with the following betting analogy (e.g. Greenland, 2006): $\mathrm{e}_{0}$ is the value where one would bet equally on $\mathrm{e}_{0}<\beta$ and $\mathrm{e}_{0}>\beta$, and the prior standard deviation, $\mathrm{sd}_{0}$, describes the uncertainty how narrowly or widely $\beta$ distributes around $\mathrm{e}_{0}$. $\mathrm{sd}_{0}$ can be determined through approximate quantiles of the normal distribution (e.g. $67 \%$ belief: value $\operatorname{sd}_{0}$ where one would bet $2: 1$ that $\mathrm{e}_{0}-\mathrm{sd}_{0}<\beta<\mathrm{e}_{0}$ $+\operatorname{sd}_{0} ; 95 \%$ belief: value $\operatorname{sd}_{0}$ where one would bet $20: 1$ that $\left.\mathrm{e}_{0}-2 * \operatorname{sd}_{0}<\beta<\mathrm{e}_{0}+2 * \operatorname{sd}_{0}\right)$. With increasing $\operatorname{sd}_{0}$ the distribution becomes increasingly flat. With infinite $\operatorname{sd}_{0}$ the distribution is completely flat and "uniform" and, in Bayesian terms, is called "uninformative" because it has no effect on the posterior (posterior = data contribution; e.g. Marsman \& Wagenmakers, 2017).

\section{Calculation of the posterior}

Bayes' theorem allows to obtain the posterior of $\beta, \mathrm{P}(\beta \mid$ data $)$, shortly written as:

$$
\mathrm{P}(\beta \mid \text { data })=\mathrm{P}(\text { data } \mid \beta) * \mathrm{P}(\beta) * \mathrm{c}
$$

$\mathrm{P}(\beta)$ denotes the prior, $\mathrm{P}($ data $\mid \beta)$ is the likelihood estimate from the data, $\mathrm{c}$ is only a constant that ensures that $\mathrm{P}(\beta \mid$ data $)$ is a probability distribution $\left(\int_{\beta} \mathrm{P}(\beta \mid\right.$ data $\left.)=1\right)$. Commonly, $\mathrm{P}(\beta \mid$ data $)$ is calculated with the numerical simulation method of MCMC (Gelman et al., 2013). This is convenient because it allows various kinds of priors, including skewed priors, for which there is no analytical access to $\mathrm{P}(\beta \mid$ data $)$. However, using $\mathrm{MCMC}$ requires several technical 
specifications, and the calculated posterior might depend on these. Moreover, MCMC is intended to be used for a specific prior that reflects a researcher's belief before conducting a study. To assess the sensitivity against this prior or to address reviewers' requests, a few other priors might be used as well. However, simulation methods like MCMC are unwieldy to illustrate how the posterior varies over an entire range of priors.

Considering the entire range, however, is easy if both the prior and the data estimate are normal. Then the posterior is also normal: $\mathrm{P}(\beta \mid$ data $) \sim \mathrm{N}\left(\mathrm{e}_{\mathrm{p}}, \mathrm{sd}_{\mathrm{p}}\right)$. With $\mathrm{e}_{\text {data }}$ and $\mathrm{sd}_{\text {data }}$ denoting the point estimate and the standard error from the data, the expectation and variance of the normal posterior, $\mathrm{e}_{\mathrm{p}}, \mathrm{sd}_{\mathrm{p}}$, can be directly calculated as (Gelman et al., 2013; Greenland, 2006):

$(*)$

$$
e_{p}=\frac{\frac{e_{0}}{s d_{0}^{2}}+\frac{e_{o b s}}{s d_{o b s}^{2}}}{\frac{1}{s d_{0}^{2}}+\frac{1}{s d_{o b s}^{2}}}, s d_{p}^{2}=\frac{1}{\frac{1}{s d_{0}^{2}}+\frac{1}{s d_{o b s}^{2}}}
$$

\section{Regions of evidence}

According to the common norm of confirmation, a target claim is said to be confirmed by evidence (at the level of $1-\alpha$ ), for instance for $\beta>0$, if $\mathrm{P}(\beta>0 \mid$ data) $>1-\alpha$. A (transparent) threshold has been argued to make researchers accountable when communicating evidence (Mayo, 2018). Since it is popular to misunderstand the frequentist p-value as 1 - the probability for a claim (often the alternative statistical hypothesis) to be true (Greenland et al., 2016b), one may assume that this probability is often what researchers wish to obtain.

For all target claims the common z-statistics is used: 


$$
\mathrm{z}_{\mathrm{p}}=\mathrm{e}_{\mathrm{p}} / \mathrm{sd}_{\mathrm{p}}
$$

$z_{p}$ has a standard deviation of 1 and, if $\beta$ equals 0 , an expectation of 0 (then it is standard normally distributed).

Evidence for $\beta>0$

There is evidence for $\beta>0$ (e.g. positive slope or positive group difference), if $\Phi\left(z_{p}\right)>1-\alpha$, where $\Phi($.$) is the cumulative of the standard normal distribution (condition a).$

Evidence for $\beta>\Delta$

Evidence just for $\beta>0$ provides only a weak statement because then $\beta$ might be infinitely close to 0 and therefore below any threshold of relevance ("nullism", Greenland, 2017). Instead, a better target might be demonstrating that $\beta$ exceeds $\Delta$, a lower boundary for clinical, public health or other practical significance. There is evidence for such a claim if $\Phi\left(\left(\mathrm{e}_{\mathrm{p}}-\Delta\right) / \mathrm{sd}_{\mathrm{p}}\right)>1-\alpha($ condition b)

Evidence for $|\beta|<\Delta$

To show that the magnitude of $\beta$ can be neglected (e.g. that two treatments are "equivalent" in their effect; Christensen, 2007) one needs to demonstrate that $\mathrm{P}(|\beta|<\Delta)=\Phi\left(\mathrm{e}_{\mathrm{p}}+\Delta / \mathrm{sd}_{\mathrm{p}}\right)$ $\Phi\left(\mathrm{e}_{\mathrm{p}}-\Delta / \mathrm{sd}_{\mathrm{p}}\right)>1-\alpha$ (condition $\left.\mathbf{c}\right)$.

\section{Calculating the regions}

After data analysis and calculating $\mathrm{e}_{\text {data }}$ and $\mathrm{sd}_{\mathrm{data}}$, the method aims to map all pairs of values 
$\left(\mathrm{e}_{0}, \mathrm{sd}_{0}\right)$ that are supported by evidence against those that are not, the regions of evidence against the region of no evidence. $\mathrm{sd}_{0}$ is chosen as it has, unlike the variance, the same unit as $\mathrm{e}_{0}$. If it is possible to take $\mathrm{e}_{\text {data }}$ and $\mathrm{sd}_{\text {data }}$ from a published paper, a recipient can determine whether a claim stands her or his own prior.

If a border function could be determined that separates the regions, mapping the regions would be straightforward. To obtain it, one could insert $\left(^{*}\right)$ in the respective condition and solve the resulting equation for $\mathrm{sd}_{0}$. Although this is mathematically possible as verified with the AI software WolframAlpha (Wolfram Alpha LLC, 2020), the solution yet for $\beta>0$ involves a complex case distinction that is unwieldy for implementation. With current computers, however, it is easy to determine the regions through simulation based on data sets with a million ( $\left.\mathrm{e}_{0}, \mathrm{sd}_{0}\right)$ points (default setting in the software described below). For this, each point $(*)$ is evaluated against the respective condition $(\mathrm{a}-\mathrm{c})$. The regions then are represented with different colours. Note that the precision in determining the exact boundary can be increased to any extent by increasing the number of simulation points (see the implementation below).

\section{Reverse Bayes and sufficiently sceptical prior}

Visualizing which priors would have supported a claim after seeing the data and circumvents the issue of subjectivity in prior choice just by showing all possibilities. It then invites for discussion on the "plausibility" and "credibility" on different priors that would lead to the one result or the other (Good, 1950, p. 80 ff.; Greenland, 2006; Held 2020; Matthews, 2001). Particularly, the sufficiently sceptical prior has been recommended to assess how robust a claim is to scepticism. This prior chooses $\mathrm{e}_{0}=0$ assuming no effect ("sceptical") and looks for the smallest $\operatorname{sd}_{0}$ ("sufficiently") that supports a claim. By doing so, it indicates how certain one may be that the true $\beta$ is in the range of 0 . For a normal prior and a normal 
estimate, $\mathrm{sd}_{0}$ can be directly calculated (Held, 2020 and references therein). If a normal prior with infinite $\operatorname{sd}_{0}$ is chosen the frequentist $p$-value for $\beta>0$ (or $\left.\beta>\Delta\right)$ equals $\mathrm{P}(\beta>0)(\mathrm{P}(\beta>$ 4)) (Marsman \& Wagenmakers, 2017). Hence, if a claim does not meet the usual frequentist evidence criterion of $\mathrm{p}<\alpha$ it does not stand any sceptical prior in Bayesian terms.

\section{Regions of prior-data conflict}

It is sometimes recommended to assess whether the data result is in line with the prior, whether the difference between these distributions exceeds chance level and, in this sense, indicates a prior-data conflict (Held, 2020). In the normal case, this is assessed with the twotailed z-statistics

$$
\mathrm{z}_{\text {conflict }}=\left(\mathrm{e}_{\text {data }}-\mathrm{e}_{0}\right) / \sqrt{ }\left(\mathrm{sd}_{\text {data }}{ }^{2}+\mathrm{e}_{0}^{2}\right)
$$

and there is a conflict at the $1-\alpha$ level if $\left|Z_{\text {conflict }}\right|>\Phi(1-\alpha / 2)$. This region can be added to a graph.

\section{Hypothetical example}

We illustrate the method with the following hypothetical example. The numbers are chosen and varied so that some important features of the method can be demonstrated. Suppose two randomly assigned groups with $\mathrm{n}=30$ each, one receives a training claimed to increase IQ test kills, the other receives no training. Outcome is the performance on a later IQ test. In the trained group the outcome was randomly drawn from a $\mathrm{N}(105,15)$ distribution and in the non-trained group from a $\mathrm{N}(100,15)$ distribution. Target parameter $\beta$ is the difference between these expectations (true $\beta=5.0$ ), and three target claims are evaluated at $\alpha=.05: \beta>$ $0, \beta>3.75(=1 / 4 \mathrm{sd}$ on the IQ scale $)$ and $|\beta|<3.75$. 
In the simulated sample those with training score $e_{\text {data }}=6.0$ IQ points higher $($ mean $=107.0$, $\mathrm{sd}=16.3 \mathrm{vs} .101 .0, \mathrm{sd}=14.2)$. The standard error of this estimate in linear regression is $\mathrm{sd}_{\mathrm{data}}$ $=3.9$, and the one-sided $p$-value for $\beta>0$ equals .068 .

For the following figures a million data points (priors, $\left.\left(\mathrm{e}_{0}, \mathrm{sd}_{0}\right)\right)$ were simulated, the $\mathrm{z}$ statistics $\mathrm{Z}_{\mathrm{p}}$ was calculated as in $(*)$, and each point was evaluated against the respective condition $(\mathrm{a}-\mathrm{c})$.

Figure 1 illustrates the regions of evidence both for $\beta>0$ and $\beta>3.75$. It shows that $\beta>0$ can be inferred with various priors that favour a positive effect, $\mathrm{e}_{0}>0$. With increasing $\mathrm{e}_{0}$ the standard deviation $\mathrm{sd}_{0}$ may be chosen higher. Small positive $\mathrm{e}_{0}$ in combination with very small $\operatorname{sd}_{0}$ allow the claim because such values express the almost certain prior belief in the claim with a minor impact of the data result on the posterior. In accordance with the frequentist one-tailed $\mathrm{p}$-value of .068 , a flat prior (approximated by choosing any $\mathrm{e}_{0}$ and a huge $\left.\mathrm{sd}_{0}\right)$ does not confirm the claim, as indicated, for example, by the out-of-region point $(0$, 12). Likewise, $\beta>0$ is not supported with any negative $\mathrm{e}_{0}$.

As it must be the case, the stronger claim $\beta>3.75$ requires a stronger prior belief in positive effects. Within the displayed range of $\mathrm{e}_{0} \leq 10, \mathrm{sd}_{0}$ must be smaller than $\mathrm{sd}_{0 \text { obs }}$ and thus dominate the data contribution. 


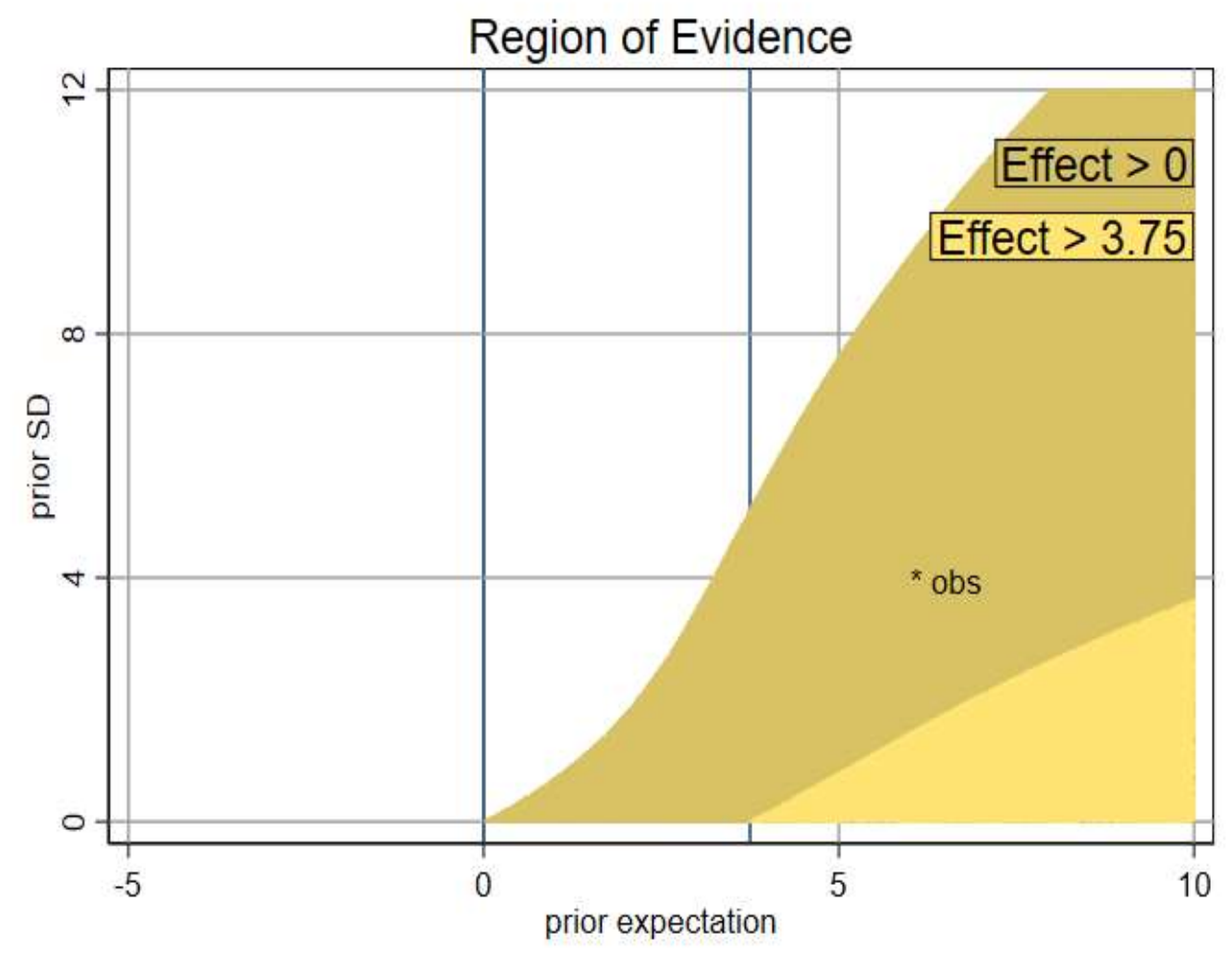

Figure 1: Regions of evidence for $\beta>0$ and $\beta>3.75$ with $e_{\text {data }}=6.0$ and $s d_{\text {data }}=3.9$.

Now suppose that the same data is evaluated against claiming that $\beta$ was neglectable small, $|\beta|<3.75$. Figure 2 a reveals that only strong prior beliefs in such values ( $s d_{0}$ much smaller than 4) allow inferring this. Figure $2 \mathrm{~b}$ adds the regions of prior-data conflict. For this, the range of $\mathrm{e}_{0}$ is extended to show that these regions are symmetrical around $\mathrm{e}_{0}$.

(a)

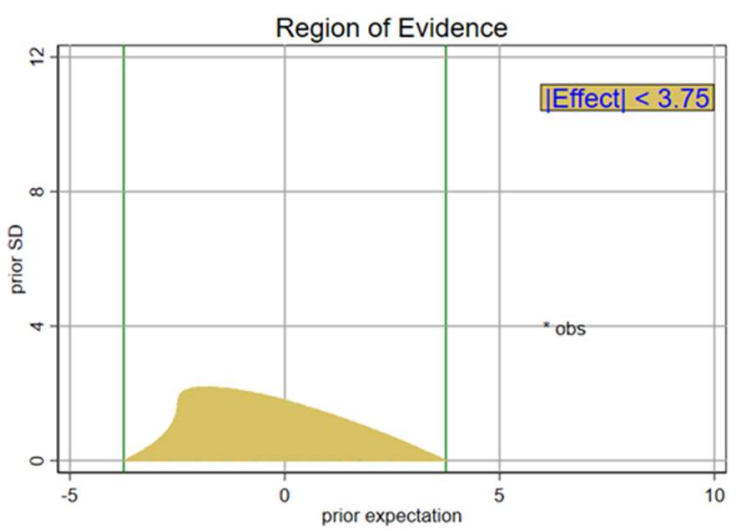

(b)

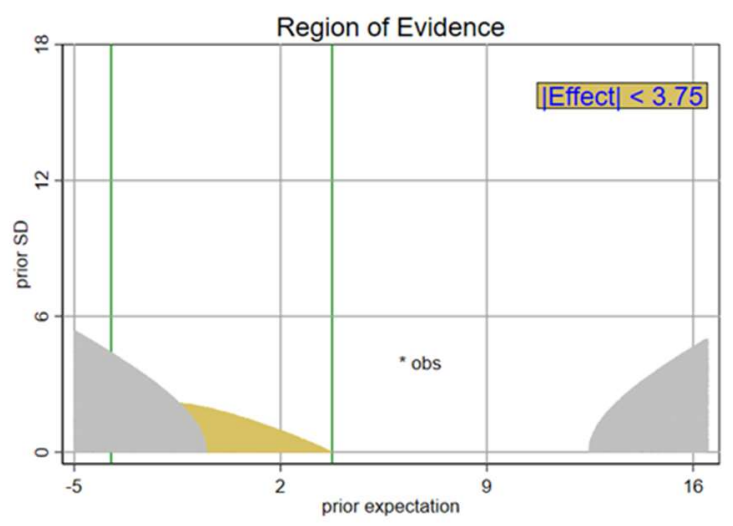


Figure 2: Regions of evidence for $|\beta|<3.75$ with $e_{\text {data }}=6.0$ and $s d_{\text {data }}=3.9$, without (a) and with (b) regions of prior-data conflict.

To illustrate the relation between regions of evidence and sufficiently sceptical priors let's go back to figure 1 , but now assume a $50 \%$ larger $\mathrm{e}_{\mathrm{obs}}$ of $9.0, \mathrm{sd}_{\mathrm{obs}}$ remaining equal. With $\mathrm{e}_{0}=0$ one obtains the minimal $\mathrm{sd}_{0}$ that allows inferring $\beta>0$ at the intersection of the vertical line $\mathrm{e}_{0}=0$ with the ochre-coloured region. The lowest such simulated $\mathrm{sd}_{0}$ equals 4.16 . (Note that this value is a bit conservative because the simulation misses points in the region with slightly lower $\mathrm{sd}_{0}$. This, however, can be addressed by simulating more points.)

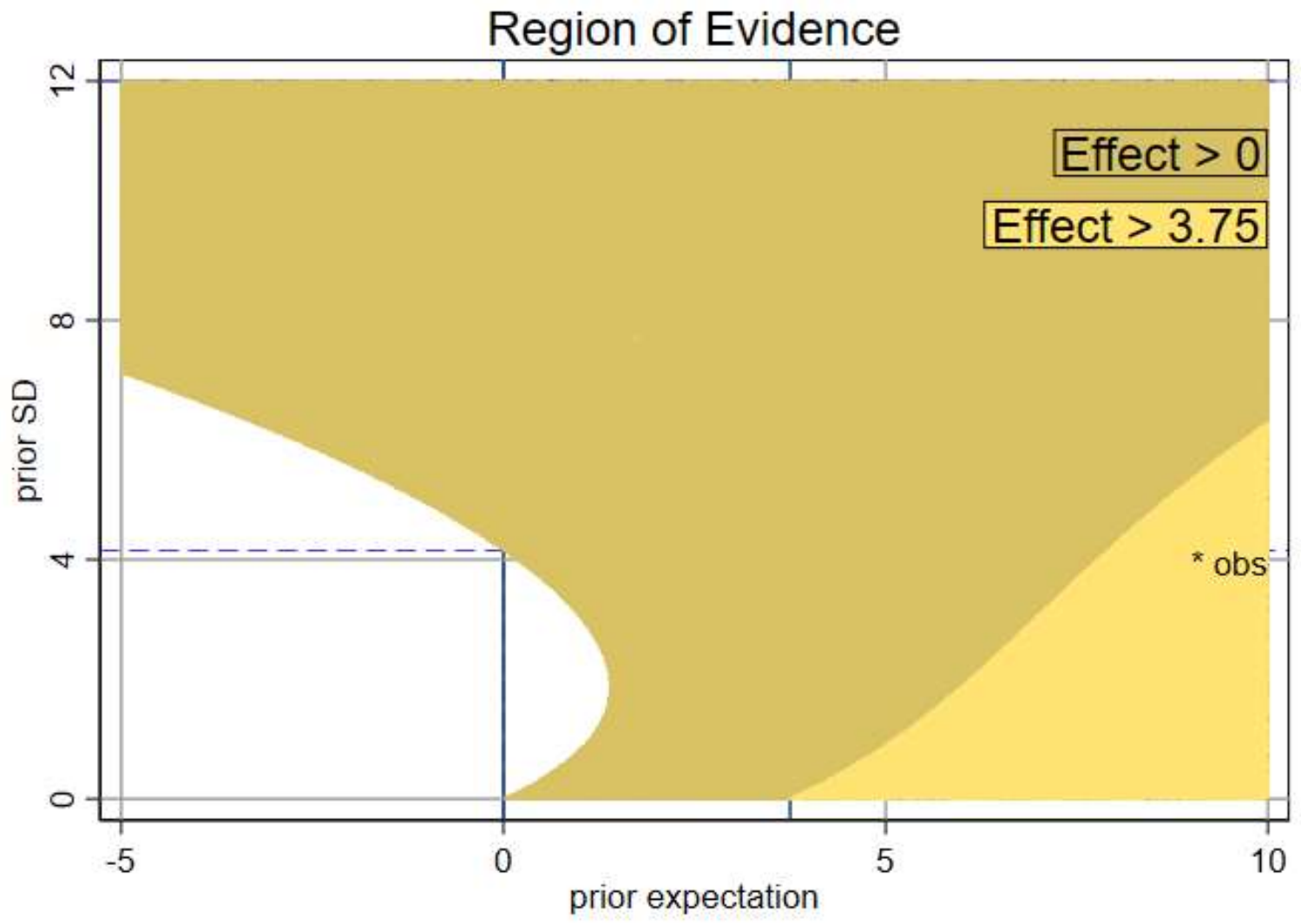

Figure 3: Regions of evidence for $\beta>0$ and $\beta>3.75$ with $e_{\text {data }}=9.0$ and $s d_{\text {data }}=3.9$. The sufficiently sceptical prior for $\beta>0$ equals $(0,4.16)$. 


\section{Forward regions of evidence}

In the posterior $(*)$ one can swap the indices 0 and obs, and the result remains unchanged. Therefore, one may use (*) also for forward considerations. Here, the prior $\left(\mathrm{e}_{0}, \mathrm{sd}_{0}\right)$ is considered as fixed and one asks what data contributions ( $\left.\mathrm{e}_{\mathrm{obs}}, \mathrm{sd}_{\mathrm{obs}}\right)$ would, if one happens to come up with these estimates, support a claim. This is useful for Bayesian sample size calculation (e.g. Lurdes et al., 2005).

Assume that one plans a study that compares two groups on the scale of $\beta=$ Cohen's d (mean difference on the standard deviation scale) and uses a sceptical prior with $\mathrm{e}_{0}=0$ and $\mathrm{sd}_{0}=$ 0.5 : expressing $95 \%$ belief in $|\beta|<1$ (approximate frequentist confidence interval in an equivalent study $=-1,1)$. With the common assumptions of normality and equal variance in both groups (and accordingly choosing equal group sizes), Figure 4 illustrates all observations ( $\mathrm{e}_{\mathrm{obs}}, \mathrm{sd}_{\mathrm{obs}}$ ) that, if observed, would support concluding $\beta>0$ or even the more informative $\beta>0.25$ (= $1 / 4$ standard deviation). It highlights the possible expectation on the data to yield at least $\mathrm{e}_{\mathrm{obs}}=0.4$, as could be suggested by the lower confidence interval limit taken from a comparable study. 


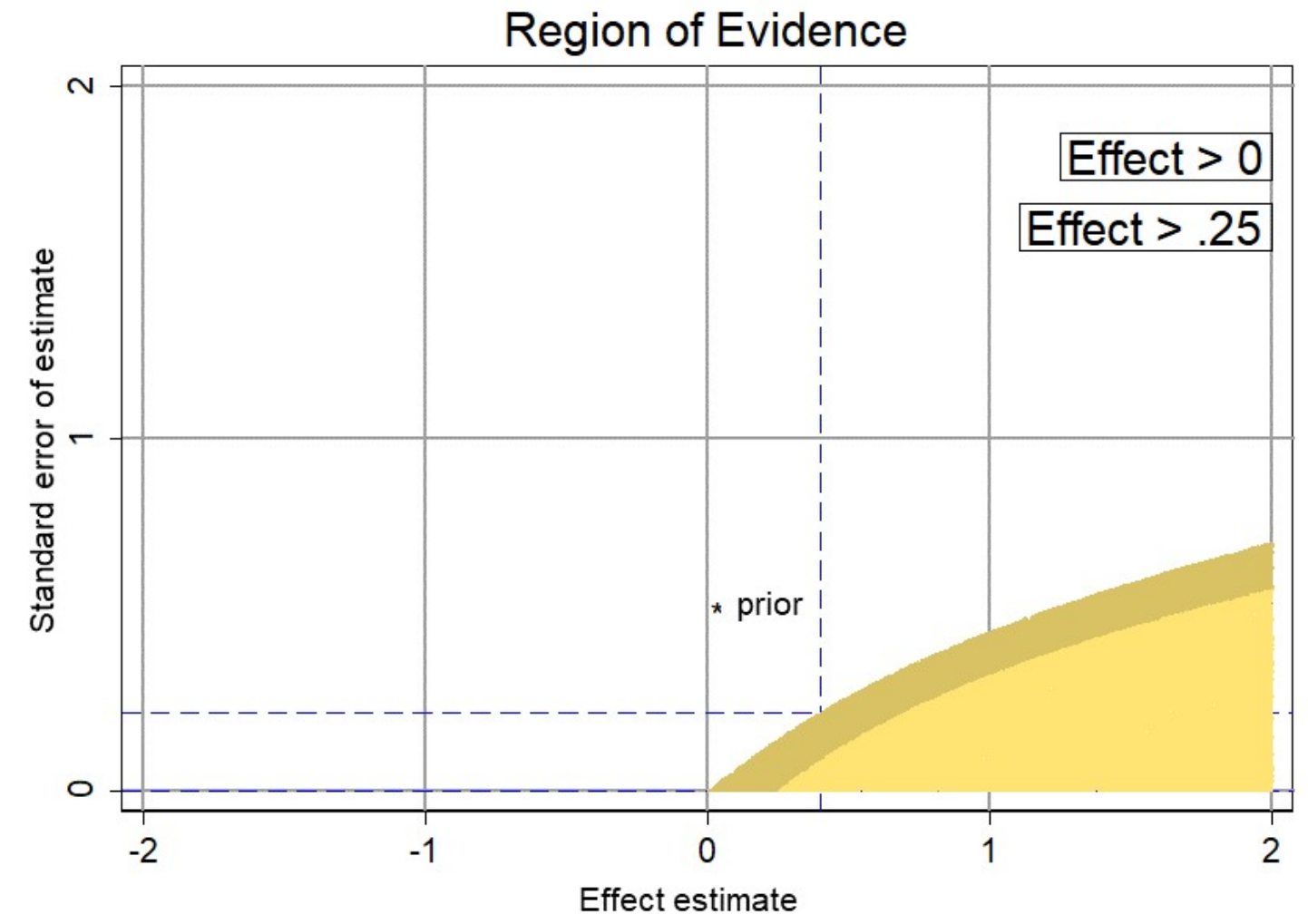

Figure 4: Forward regions of evidence that support $\beta>0$ and $\beta>0.25$, respectively, with prior $e_{0}=0$ and $s d_{0}=0.5$ showing the largest possible $s d_{o b s}$ if one is to observe $e_{o b s}=0.4$.

Then one would require an $\operatorname{sd}_{\mathrm{obs}}$ of at most 0.22 to show $\beta>0$. To demonstrate $\beta>0.25, \mathrm{sd}_{\mathrm{obs}}$ must be at most 0.08 . The relation to the required group size $n$ is $\operatorname{sd}_{\mathrm{obs}}=1 / \sqrt{ }(1 / 2 * n)$, thus $n$ $=2 / \mathrm{sd}_{\mathrm{obs}} \wedge 2$. Hence, a group size of 41 is required for $\beta>0$, but 281 for $\beta>0.25$. Note that the sceptical prior shrinks positive estimates towards 0 as necessary to convince a sceptical who would use this prior. With a flat prior one would only need $n=35$ and $n=242$, respectively.

\section{Implementation in Stata}

The figures in this article were created with a self-written Stata command called arevi.ado. It can be used after any estimation (regression) command that saves $\mathrm{e}_{\mathrm{obs}}$ and $\mathrm{sd}_{\mathrm{obs}}$ as macros. In 
its immediate mode one may assess what priors support a claim based on published results. Besides, arevi optionally returns:

- $\quad$ the posterior distribution $\left(\mathrm{e}_{\mathrm{p}}, \mathrm{sd}_{\mathrm{p}}\right)$

- a one-tailed credibility interval

- z-statistics for $\beta>0$ and $\beta>\Delta$

- $\quad$ and the associated posterior probabilities.

If $|\beta|<\Delta$ is evaluated (option "rope"), it instead returns the two-tailed z-statistics and the posterior probability of $|\beta|<\Delta$. Besides, a particular $\mathrm{e}_{0}$ might be specified to report the minimum and maximum $\operatorname{sd}_{0}$ that are in line with a claim or vice versa (with fixed $\mathrm{sd}_{0}$ ). This is done within the simulated $\mathrm{e}_{0}$ and $\mathrm{sd}_{0}$ ranges, which can be freely chosen (like the number of simulated data points, default $=1,000,000)$. Other options address the graphical output, for example, adding lines. The code of arevi.ado and a documentation ("help file") can be obtained at https://osf.io/jxnsv/.

\section{Discussion}

Region of evidence is a straightforward method that visually aids the use and interpretation of a Bayesian analysis with a normally distributed data estimate and prior. It illustrates the full range of normal priors that support a claim after analysis. This transparently integrates the prior assumptions that different researchers might have and invites for discussion on the prior's choice. Of course, such assessment is easily influenced by seeing the data results, but this seems unavoidably because otherwise all potential recipients of a result must have preregistered their priors. This drawback is so obvious that it should naturally be part of discussions. Actually, recipients may assess only the impact of potential priors that, would 
they have been chosen before seeing the data (at least without being affected by this), would now support a claim or not.

The method is restricted to normal distributions that are fully characterized by only two parameters. It builds on the convenience that the posterior can be directly calculated from the prior and the data contribution. Its use with other distributions (if the prior and a data estimate have different distributions) seems difficult with simulation methods, but not impossible. Besides, regions of evidence appear to serve some teaching aspects: They visualize the relative weights of the prior and a data estimate (through their standard deviations) and how a stronger claim (choosing a larger $\Delta$ ) modifies the regions of evidence non-linearly.

Finally, they demonstrate the use of Bayes for sample size planning with the advantages of conducting a study that is suitable to convince a sceptic (as in Figure 4) or to save resources when sound prior knowledge is available in favour of an effect.

\section{Acknowledgements}

The author wants to thank Robert Miller for his ideas in prior discussions.

\section{References}

Berger, J. O., \& Berger, J. O. (1985). Statistical decision theory and Bayesian analysis. New York: Springer-Verlag.

Carlin, B. P., \& Louis, T. A. (2000). Bayes and Empirical Bayes Methods for Data Analysis. 2nd Edition, Chapman and Hall/CRC, New York. doi: $\underline{10.1201 / 9781420057669}$ 
Christensen, E. (2007). Methodology of superiority vs. equivalence trials and non-inferiority trials. Journal of Hepatology, 46(5), 947-954. doi: 10.1016/j.jhep.2007.02.015

Depaoli, S., Winter, S. D., \& Visser, M. (2020). The Importance of Prior Sensitivity Analysis in Bayesian Statistics: Demonstrations Using an Interactive Shiny App. Frontiers in psychology, 11, 608045. doi: $\underline{10.3389 / \text { fpsyg.2020.608045 }}$

Dienes, Z. (2016). How Bayes factors change scientific practice. Journal of Mathematical Psychology, 72, 78-89. doi: 10.1016/j.jmp.2015.10.003

Fortuin, V. (2021). Priors in Bayesian Deep Learning: A Review. ArXiv, abs/2105.06868.

Gelman, A., Carlin, J. B., Stern, H. S., Dunson, D. B., Vehtari, A., \& Rubin, D. B. (2013). Bayesian Data Analysis (3nd ed.). Chapman and Hall/CRC. doi: 10.1201/b16018

Good, I. J. (1950). Probability and the Weighing of Evidence. Griffin.

Greenland, S. (2005). Multiple-bias modelling for analysis of observational data (with discussion). Journal of the Royal Statistical Society Series A (Statistics in Society) 168(2), 267-306. doi: 10.1111/j.1467-985X.2004.00349.x

Greenland, S. (2006). Bayesian perspectives for epidemiological research: I. Foundations and basic methods. International Journal of Epidemiology 35(3), 765-775, doi: $\underline{10.1093 / \mathrm{ije} / \mathrm{dyi} 312}$

Greenland, S. (2009a). Bayesian perspectives for epidemiologic research: III. Bias analysis via missing-data methods. International Journal of Epidemiology 38(6), 1662-73. doi: $\underline{10.1093 / \mathrm{ije} / \mathrm{dyp} 278}$ 
Greenland, S. (2009b). Relaxation Penalties and Priors for Plausible Modeling of Nonidentified Bias Sources. Statistical Science 24(2), 195 - 210. doi: $\underline{10.1214 / 09-S T S 291}$

Greenland, S. (2012). Causal inference as a prediction problem. In: Berzuini, C., Dawid, P., \& Bernardinelli, L. (eds). Causality, Statistical Perspectives and Applications. Hoboken, NJ: Wiley, p. 44-52.

Greenland, S. (2017). Invited Commentary: The Need for Cognitive Science in Methodology, American Journal of Epidemiology 186(6), 639-645, doi: $\underline{10.1093 / \mathrm{aje} / \mathrm{kwx} 259}$

Greenland, S., Mansournia, M. A., \& Altman, D. G. (2016a). Sparse data bias: a problem hiding in plain sight. $B M J$ 27(352), i1981. doi: $\underline{10.1136 / \mathrm{bmj} . \mathrm{i} 1981}$

Held, L. (2020). A new standard for the analysis and design of replication studies. Journal of the Royal Statistical Society 183(2), 431-448 doi: 10.1111/rssa.12493

Höfler, M., Venz, J., Trautmann, S., \& Miller, R. (2018). Writing a discussion section: how to integrate substantive and statistical expertise. BMC Medical Research Methodology 18(34). doi: $\underline{10.1186 / \mathrm{s} 12874-018-0490-1}$

Lurdes, Y. T. I., Berry, D. A., \& Parmigiani, G. (2005). Relationship Between Bayesian and Frequentist Sample Size Determination. The American Statistician, 59(1)79-87, doi: $\underline{10.1198 / 000313005 \times 21069}$

Maclure, M., \& Schneeweiss, S. (2001). Causation of bias: the episcope. Epidemiology 12(1),114-22. doi: 10.1097/00001648-200101000-00019. 
Marsman, M., \& Wagenmakers, E.-J. (2017). Three insights from a Bayesian interpretation of the one-sided P value. Educational and Psychological Measurement, 77(3), 529539. doi: $\underline{10.1177 / 0013164416669201}$

Matthews, R. A. J. (2001). Why should clinicians care about Bayesian methods (with discussion)? Journal of Statistical Planning and Inference 94, 43-71. doi: $\underline{10.1016 / \mathrm{S} 0378-3758(00) 00235-4}$

Mayo, D. G. (2018). Statistical Inference as Severe Testing: How to Get Beyond the Statistics Wars. Cambridge: Cambridge University Press. doi: 10.1017/9781107286184

O'Hara, R. B., \& Sillanpää, M. J. (2009). A review of Bayesian variable selection methods: what, how and which. Bayesian Analysis 4(1), 85-117. doi: 10.1214/09-BA403

Severini, T. A. (2000). Likelihood Methods in Statistics. Oxford University Press.

Vandekerckhove, J., Rouder, J. N., \& Kruschke, J. K. (2018). Editorial: Bayesian methods for advancing psychological science. Psychonomical Bulletin \& Review 25, 1-4. doi: $\underline{10.3758 / \mathrm{s} 13423-018-1443-8}$

Wagenmakers, E.-J., Marsman, M., Jamil, T., Ly, A., Verhagen, J., Love, J., Selker, R., Gronau, Q. F., Šmíra, M., Epskamp, S., Matzke, D., Rouder, J. N., \& Morey, R. D. (2018). Bayesian inference for psychology. Part 1: Theoretical advantages and practical ramifications. Psychonomic Bulletin \& Review, 25(1), 35-57. doi: $\underline{10.3758 / \mathrm{s} 13423-017-1343-3}$

Wolfram Alpha LLC (2020). Wolfram | Alpha. WolframAlpha. [,,solve[((x/y^2+e/s^2)/ $\left.\left(1 / y^{\wedge} 2+1 / s^{\wedge} 2\right)\right) /\left(\left(1 /\left(1 / y^{\wedge} 2+1 / s^{\wedge} 2\right)\right)^{\wedge} 1 / 2\right)>p, p>0, s>0, y>0, y^{6}$, „https://www.wolframalpha.com/input/?i=solve $\% 5 \mathrm{~B} \% 28 \% 28 \mathrm{x} \% 2 \mathrm{Fy} \% 5 \mathrm{E} 2+\% 2 \mathrm{~B}+\mathrm{e} \%$ 
2Fs $\% 5 \mathrm{E} 2 \% 29+\% 2 \mathrm{~F}+\% 281 \% 2 \mathrm{Fy} \% 5 \mathrm{E} 2+\% 2 \mathrm{~B}+1 \% 2 \mathrm{Fs} \% 5 \mathrm{E} 2 \% 29 \% 29+\% 2 \mathrm{~F} \% 28 \% 281$ $\% 2 \mathrm{~F} \% 281 \% 2 \mathrm{Fy} \% 5 \mathrm{E} 2+\% 2 \mathrm{~B}+1 \% 2 \mathrm{Fs} \% 5 \mathrm{E} 2 \% 29 \% 29 \% 5 \mathrm{E} 1 \% 2 \mathrm{~F} 2 \% 29+\% 3 \mathrm{E}+\mathrm{p} \% 2 \mathrm{C}+\mathrm{p}$ \%3E0\%2C+s\%3E0\%2C+y \%3E0\%2C+y $\% 5$ D"] Retrieved dec 8th, 2020. 